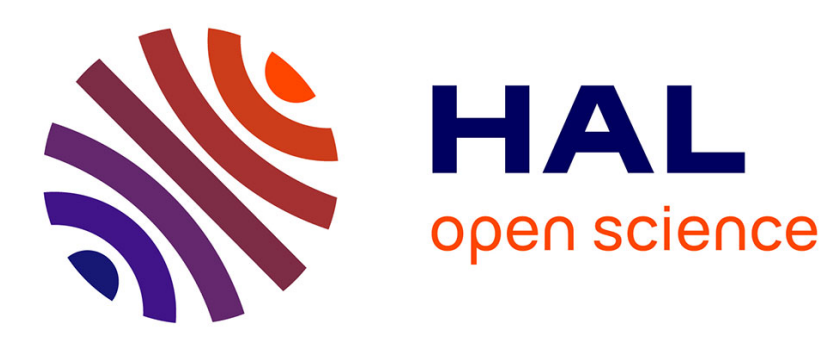

\title{
Internal Friction Peaks at Low Temperatures : a Kink Model Analysis
}

\author{
G. Costantini, F. Marchesoni
}

\section{To cite this version:}

G. Costantini, F. Marchesoni. Internal Friction Peaks at Low Temperatures: a Kink Model Analysis. Journal de Physique IV Proceedings, 1996, 06 (C8), pp.C8-187-C8-190. 10.1051/jp4:1996838 . jpa00254646

\section{HAL Id: jpa-00254646 https://hal.science/jpa-00254646}

Submitted on 1 Jan 1996

HAL is a multi-disciplinary open access archive for the deposit and dissemination of scientific research documents, whether they are published or not. The documents may come from teaching and research institutions in France or abroad, or from public or private research centers.
L'archive ouverte pluridisciplinaire HAL, est destinée au dépôt et à la diffusion de documents scientifiques de niveau recherche, publiés ou non, émanant des établissements d'enseignement et de recherche français ou étrangers, des laboratoires publics ou privés. 


\title{
Internal Friction Peaks at Low Temperatures: a Kink Model Analysis
}

\author{
G. Costantini and F. Marchesoni
}

I.N.F.N., VIRGO-Project, Università di Perugia, Perugia, Italy

Dipartimento di Matematica e Fisica, Università di Camerino, Camerino, Italy

\begin{abstract}
Two internal friction peaks discovered in pure aluminum at increasingly low temperatures, respectively by Lax and Filson and by Kosugi and Kino, are interpreted as different occurrences of the very same mechanism responsible for the better-known Bordoni peak, namely the nucleation of thermal kink pairs.
\end{abstract}

Most f.c.c. metals subjected to cold work develop a number of peaks in the curve of internal friction (IF) versus temperature [1,2]. The most prominent maximum (Bordoni peak [3]) and its subsidiary (Niblett-Wilks peak [2]) were detected first almost fifty years ago in the range $50-100 \mathrm{~K}$. Since then, a variety of less pronounced peaks were discovered both at higher (Hasiguti peaks $[1,4]$ ) and lower temperatures. Two types of IF peaks have been reproduced systematically (mostly in pure aluminum) by lowering the temperature, namely the Lax-Filson (LF) [5] and the Kosugi- Kino (KK) peak [6]. Most properties of these peaks resemble those detailed for the Bordoni peak [1-3,7].

The Bordoni peak is currently interpreted $[1,2]$ as the signature of one or more relaxation processes taking place in the lattice dislocation network [8]. A dislocation segment which lies along a Peierls valley can overcome the relevant confining barriers (quantified by the Peierls stress $\sigma_{P}$ ) only by nucleating a kink-antikink pair. Nucleation is a thermally activated phenomenon $[9,10]$ and contributes to IF a Debye peak with frequency constant given by the nucleation rate in the dislocation segment. The kink model of the Bordoni peak was introduced first by Seeger and Schiller [11] and then refined to account for the emerging complexity of the experimental description [12,13]. More recently, the kink model was extended to explain the onset of the Hasiguti peaks [4], as well.

Following Ref. [9], we represent the position of a dislocation line on its glide plane by a sine-Gordon (SG) string $\phi(x, t)$ coupled to a heat-bath,

$$
\phi_{t t}-c_{0}^{2} \phi_{x x}+\omega_{0}^{2} \sin \phi=-\alpha \phi_{t}+\zeta(x, t)
$$

where $c_{0}$ and $\omega_{0}$ are the parameters of the unperturbed SG equation, the sinusoidal potential $-\omega_{0}^{2} \cos \phi$ models a system of parallel Peierls valleys, $\alpha$ denotes the damping constant and the gaussian noise $\zeta(x, t)$ has zero mean and correlation $\left\langle\zeta(x, t) \zeta\left(x^{\prime}, t^{\prime}\right)\right\rangle=2 \alpha k T \delta\left(t-t^{\prime}\right) \delta\left(x-x^{\prime}\right)$ [15].

The Lax-Filson peak. - A lattice defect in the immediate vicinity of the dislocation line acts as a pinnng point. We represent the interaction of the dislocation $\phi(x, t)$ with a single pinner (at a distance $r$, perpendicularly to $\phi$ at $x=0$ ) by means of the local force term [8]

$$
F(x)=-\left(U_{C} / a\right) \delta(x / a)
$$

where $U_{C}=U_{C}(r)$ is the Cottrell pinning energy. Moreover, the pinners are uniformly distributed in the $x$ direction and tend to concentrate in the vicinity to the dislocation core. 
A dislocation segment lying along one Peierls valley, say $\phi_{0}=0$, is unstable against the nucleation of thermal kink-antikink pairs $[11,16]$. Thermal fluctuations, $\zeta(x, t)$ in eq $(1)$, trigger the process by activating a critical nucleus, the length of which decreases with increasing the intensity of the external driving term [10]. In the present case the external drive is provided by the local pinning force $F(x)$ in eq(2). At higher temperatures, the driving action exerted by the pre-existing kinks and antikinks dominates [9], whence the Bordoni relaxation model of Ref. [13]. A large nucleus $\phi_{N}(x, X)$ with length $2 X$ is well reproduced by the linear superposition of a kink and an antikink centered at $\mp X$, respectively,

$$
\phi_{N}(x, X)=\phi_{+}(x+X, 0)+\phi_{-}(x-X, 0)=4 \tan ^{-1}(\sinh X / \cosh x)
$$

where $\phi_{ \pm}(x, t)=4 \tan ^{-1} \exp ( \pm \beta[x-X(t)] / d),(\bmod 2 \pi)$, is the kink (antikink) solution of the unperturbed SG equation (1) for $\alpha=0$, with center of mass $X(t)=x_{0}+u t$ and width $d=c_{0} / \omega_{0}$. At low temperatures the relativistic boost factor $\beta=1 / \sqrt{1-u^{2} / c_{0}^{2}}$ tends to unity [9]. The $\phi_{ \pm}$components of $\phi_{N}$ can be treated as quasi-particles with coordinates $\mp X$ and mass $M_{0}=E_{0} / c_{0}^{2}=8 / d$. They experience two contrasting forces: an attractive force due to the vicinity of the nucleating partner and a repulsive force due to the external drive (2). The potential function corresponding to the internal force is approximated by [17] $V_{K}(X)=-E_{0} / \cosh (2 X / d)$. The effective potential generated by a pinner centered at the mid-point of $\phi_{N}, X=0$, can be determined by means of a global energy-balance argument $[18,19]$,

$$
V_{F}(X)=\left(U_{C} / 2 \pi\right) \phi_{N}(-X, 0)=-\left(2 U_{C} / \pi\right) \tan ^{-1}[\sinh (X / d)]
$$

The total potential $V_{N}(X)=V_{K}(X)+V_{F}(X)$ describes the decay of the nucleus $\phi_{N}$ in terms of the pair coordinate $X$. The pair nucleation rate in a damped $S G$ chain reads

$$
\Gamma=\frac{\left|\lambda_{0}^{N}\right|}{2 \pi} \frac{Z_{N}}{Z_{0}} \exp \left(-\Delta E_{N} / k T\right)
$$

Here, $Z_{0}$ and $Z_{N}$ denote the partition function of the $\phi_{0}$ and $\phi_{N}$ field configurations, respectively. A standard calculation yields [19] $Z_{N} / Z_{0}=8\left(\Delta E_{N} / k T\right)^{1 / 2}\left(\omega_{0}^{2} / 2 \pi\left|\lambda_{0}^{N}\right|\right)^{1 / 2}$ The activation energy $\Delta E_{N}=V_{N}\left(X_{b}\right)-V_{N}\left(X_{0}\right)$ and the negative eigenvalue $\lambda_{0}^{N}$ corresponding to the decay mode of $\phi_{N}\left[X_{0}\right.$ and $X_{b}$ locate the local minimum and the barrier of the potential $V_{N}(X)$ ], are immediately obtainable from the analytical expression of $V_{N}(X)$. For $U_{C}=0.1 e V$, the activation energy $\Delta E_{N}$ in pure aluminum is $0.023 \mathrm{eV}$, in close agreement with the experimental value $0.024 \mathrm{eV}$ reported in Ref. [5]. Analogously, in pure copper $\Delta E_{N} \leq 0.01 \mathrm{eV}$, thus making the LF peak detectable at much lower temperatures, only. This result explains why the evidence for LF peak(s) in copper is not firmly established, yet.

We make now a few important remarks:

(i) Since the impurity assisted nucleation mechanism is local by definition, no side-peak ought to be associated with a LF peak (at variance with the Bordoni mechanism, where the NiblettWilks peak signals the interaction of the nucleating pair with the pre-existing geometrical kinks (antikinks) [13]) and the LF peak is expectedly narrower than the Bordoni peak;

(ii) The height of the LF peak $\left(\Delta_{L F}\right.$ at temperature $\left.T_{L F}\right)$ and of the Bordoni peak $\left(\Delta_{B}\right.$ at temperature $\left.T_{B}\right)$ are of the same order. In view of Ref. [13], $\Delta_{L F} / \Delta_{B}=\left(T_{B} / T_{L F}\right)\left[n_{i m p} / n\left(T_{B}\right)\right]$, where $n\left(T_{B}\right)$ is the equilibrium (anti)kink density, $n(T)=\left(2 E_{0} / \pi k T d^{2}\right)^{1 / 2} \exp \left(-E_{0} / k T\right)[8-$ 11 , and $n_{i m p}$ is the linear density of the pinners acting on the dislocation line. A rough estimate for $n_{i m p}$ reads $n_{i m p} \equiv 1 / l_{i}=c_{i} N_{a}\left(\pi b^{2}\right) \Omega_{\Lambda}$, where the volume impurity density (defined as the impurity atom concentration $c_{i}$ times the number of metal atoms per volume unit $N_{a}$ ) has been integrated over the dislocation effective cross-section $\pi b^{2}$. The geometrical factor $\Omega_{\Lambda}$ for dislocations in the glide planes (111) of a f.c.c. lattice is $1 / 6$. For $6 \mathrm{~N}$ aluminum $c_{i} \simeq 10^{-6}$, $N_{a} \simeq 1.810^{23} \mathrm{~cm}^{-3}, n\left(T_{B}\right) \simeq 10^{2} \mathrm{~cm}^{-1}$ and $T_{B} / T_{L F} \sim 2 E_{0} / \Delta E_{N} \simeq 8$, whence $\Delta_{L F} / \Delta_{B} \sim 1$, 
as reported in the experimental literature $[5,6]$. The estimate of $n_{i m p}$ at extremely low impurity densities requires some caution [19].

The Kosugi-Kino peak. - The KK peak has been detected at temperatures $T_{K K}$ much lower than $T_{L F}\left(T_{K K} \sim 10 K[6]\right)$, where thermal activation over the barrier of $V_{N}(X)$ cannot occur. Moreover, in Ref. [6] it is clearly proven that the KK peak shows up also in samples of extremely pure aluminum, where impurity assisted nucleation becomes statistically irrelevant. On the other hand, the properties of the KK peak recall closely those detailed by Bordoni and co-workers [3]. This leads us to invoke a new low temperature nucleation mechanism.

A (screw or edge) dislocation line which glides on the (111) planes of a f.c.c. metallic lattice is subjected to two different stress fields. The more popular lattice field is represented by the Peierls valleys, which motivate theorists to prefer at low temperatures the kink model [11] to the vibrating string model [20]. However, to describe the crystallographic structure of the glide planes (111) more accurately, one should introduce at least another periodic structure of shallow valleys that intersect the Peierls valleys with a different spacing $\boldsymbol{a}^{\prime}$. A single kink (antikink) glides now parallel to the Peierls valleys on an undulated background [21] represented, to first a approximation, by a sinusoidal (Schottky) potential with period $a^{\prime}$ and amplitude $\sigma_{S}$ (with $\left.\sigma_{S} \ll \sigma_{P}\right)[14]$, i.e. $\sigma_{S} / G \sim-(a / d)\left(\sigma_{P} / G\right)^{2}$.

Let us suppose that, due to a static deformation, all geometrical kinks (antikinks) of a dislocation segment of length $l$ packed together against the pinning points, spaced by a minimal distance of the order of $d$. At very low temperature, nucleation of new kink-antikink pairs is inhibited. Hence, the question rises whether the dislocation segment may further bow out in the direction of the external stress. Multikink dislocation depinning, originally advocated by Alefeld [22], is not the only mechanism one can envisage to this purpose. In fact, the piled-up kinks (antikinks) are likely localized at the bottom of relatively deep Schottky valleys; moreover, they repel their nearest neighbors with a potential $U_{K}$, inverse proportional to their relative distance [11]. The corresponding equation of motion for the $i$-th kink (antikink) reads $[23]$

$$
M_{0} \ddot{X}_{i}=-\alpha M_{0} \dot{X}_{i}-2 \pi F_{S} \sin \left(2 \pi X_{i} / a^{\prime}\right)-\left(d U_{K} / d X_{i}\right)+\eta_{i}(t)
$$

where $U_{K}=V_{K} \sum_{i=1}^{n_{l}}\left|X_{i}-X_{i-1}\right|^{-1}$ with $X_{0}=0$. Here, $X_{i}$ is the center of mass of the $i-$ th kink (antikink), $n_{l}$ is the number of packed geometrical kinks (antikinks), $F_{S}=a^{\prime} b \sigma_{S} / 2 \pi$, $V_{K}=a^{3} C / 4 \pi$ (see Refs. [11] and [15]) and $\eta_{i}(t)$ are gaussian, zero-mean valued noises with correlation functions $\left\langle\eta_{i}(t) \eta_{j}(0)\right\rangle=2 M_{0} \alpha k T \delta_{i j} \delta(t)$.

For application purposes, we set $a=a^{\prime}$ and remember that $d \gg a$, so that expanding $U_{K}$ around $\left|X_{i}-X_{i-1}\right|=d$ yields $d U_{K} / d X_{i}=c_{s}^{2}\left(X_{i+1}-X_{i}\right)+c_{s}^{2}\left(X_{i-1}-X_{i}\right)$ with $c_{s}^{2}=C a^{3} / d^{3}$. Thus, the coordinates of the $n_{l}$ kinks (antikinks) obey a discretized SG equation. Now, the dislocation segment may further bow out by pushing one geometrical kink (antikink) over a. Schottky barrier. This happens as a collective phenomenon, where pairs of superkinksantikinks are nucleated, each involving a rather large number of individual kinks (antikinks). The analytical description of such a nucleation mechanism is exactly the same as in the revised theory [13] of Seeger and Schiller nucleation. In particular, the corresponding activation energy $\Delta E_{S}$ is of the order of $2 E_{S}$, where $E_{S}$ is the rest energy of a superkink (antikink). Since $\omega_{S}^{2} / \omega_{0}^{2}=\sigma_{S} d / \sigma_{P} a, c_{S}^{2} / c_{0}^{2}=(a / d)^{3}$ and $E_{S}=8 \omega_{S} c_{S}$, it follows that

$$
\Delta E_{S} / \Delta E_{B}=E_{S} / E_{0}=(a / d) \sqrt{\sigma_{S} / \sigma_{P}}
$$

On making use of the following estimates for the aluminum lattice parameters, $\sigma_{S} \sim 10^{-2} \sigma_{P}$ and $d \sim 4 a$, it turns out that $\Delta E_{S} / \Delta E_{B} \simeq 2.510^{-2}$, in close agreement with the experimental results reported in Refs. [6,7]. Note that the predicted superkink (antikink) length $d_{S}=c_{S} / \omega_{S}$ is of the order of $a\left(\Delta E_{B} / \Delta E_{S}\right) \simeq 40 a$, at variance with the estimate of $600 a$ obtained in Ref. [6] by means of Seeger and Schiller theory [11]. In copper, a KK peak is expected for much lower temperature and higher purity than in aluminum [19]. 
Note that the LF and KK peaks are clearly distinguishable by varying the impurity atom concentration $c_{i}$. The KK peak occurs for any sample purity with $c_{i}<b / m_{p} L_{N}$, where $L_{N}=1 / \sqrt{\Lambda}$ is the maximum dislocation segment length in a lattice with dislocation density $\Lambda$ (dislocation length per volume unit) [6]. The impurity-assisted nucleation mechanism works for impurity atom concentrations ranging between $c_{m} \simeq b / L_{N}$ and $c_{M} \simeq b / m_{p} d$ [19]. For the aluminum lattice parameters introduced above and $\Lambda \simeq 10^{4} \mathrm{~cm}^{-2}$, we conclude that the $\mathrm{LF}$ peak is observable for $10^{-4} \leq c_{i} \leq 10^{-2}$, only, in agreement with Refs. [5-7, 24].

\section{References}

[1] Nowick A. S. and Berry B. S., Anelastic Relaxation in Crystalline Solids (Academic, New York, 1972)

[2] Niblett D. H., in Physical Acoustics, W. P. Mason ed., Vol IIIA (Academic, New York, 1966), p. 77.

[3] Bordoni P. G., Ricerca Sci. 19 (1949) 856.

[4] Marchesoni F., Appl. Phys. Lett. 67 (1995) 217.

[5] Lax E. and Filson D. H., Phys. Rev. 114 (1959) 1273.

[6] Kosugi T. and Kino T., Materials Sci. Forum 119-121 (1993) 177 and J. Phys. Soc. Jpn. 58 (1989) 4269.

[7] Gremaud G., Bujard M. and Benoit W., J. Appl. Phys. 61 (1987) 1795; Bujard M., Gremaud G. and Benoit W., J. Appl. Phys. 62 (1987) 3173.

[8] Nabarro F. R. N., Theory of Crystal Dislocations (Dover, New York, 1987)

[9] Marchesoni F., Phys. Rev. Lett. 73 (1994) 2394; Hänggi P., Marchesoni F. and Sodano P., Phys. Rev. Lett. 60 (1988) 2563.

[10] Marchesoni F., Ber. Bunsenges. Phys. Chem. 95 (1991) 353.

[11] Seeger A. and Schiller P., in Physical Acoustics, W. P. Mason ed., Vol IIIA (Academic, New York, 1966), p. 361.

[12] Pare' V. K., J. Appl. Phys. 32 (1961) 332.

[13] Marchesoni F., Phys. Rev. Lett. 74 (1995) 2973.

[14] Schottky G., Phys. Stat. Sol. 5 (1964) 697.

[15] to make contact with a real dislocation, lattice units must be restored in eq(1), i.e. $\phi \rightarrow 2 \pi \phi / a, c_{0}^{2} \rightarrow C / \pi \rho a^{2}, \omega_{0}^{2} \rightarrow 2 \sigma_{P} b / \rho a^{3}, \alpha \rightarrow B / \pi \rho a^{3}$ and $F(x, t) \rightarrow a b \sigma(x, t) / 2 \pi$, where $a$ is the lattice constant, $b$ is the Burgers vector, $\rho$ is the density of the material, $B$ and $C \simeq G b^{2}$ are the viscous and tension constants, $G$ is the shear modulus and $\sigma(x, t)$ is an external, time-dependent stress field.

[16] Hirth J. P. and Lothe J., Theory of Dislocations (Wiley, New York, 1982), Chap. 8.

[17] this approximation works well at large distances, i.e. for $X \gg d$. The use of a more refined expression for $V_{K}(X)$ (see eq(17) of Ref. [10]) changes our subsequent analytical extimates marginally, only.

[18] McLaughlin D. W. and Scott A. C, Phys. Rev. A18 (1978) 1652.

[19] Marchesoni F., J. Appl. Phys. 80 (1996) to be published.

[20] Granato A. V. and Lücke K., in Physical Acoustics, W. P. Mason ed., Vol IVA (Academic, New York, 1966), p. 225.

[21] Brailsford A. D., Phys. Phys. 122 (1961) 778 and 128 (1962) 1033.

[22] Alefeld G., Phil. Mag. 11 (1965) 809.

[23] Marchesoni F. and Patriarca M., Phys. Rev. Lett. 72 (1994) 4101.

[24] Lauzier J., Hillairet J., Gremaud G. and Benoit W., J. Phys. C2 (1990) 9247. 